\title{
DESARROLLO, REESTRUCTURACIÓN DEL GASTO PÚBLICO Y ALIANZAS PÚBLICO-PRIVADAS*
}

Jesús Botero García

José García Guzmán

* DOI: https://doi.org/10.18601/01245996.v20n38.08. Este artículo es el resultado de la investigación Política fiscal en economías emergentes, realizada para la Universidad EAFIT. Los autores agradecen los comentarios, sugerencias y aportes del profesor Carlos Esteban Posada. Recepción: 2609-2016, modificación final: 22-01-2018, aceptación: 19-02-2018. Sugerencia de citación: Botero G., J. y García G., J. (2018). Desarrollo, reestructuración del gasto público y alianzas público-privadas. Revista de Economía Institucional, 2O(38), 185-207.

${ }^{a}$ Especialista en Política Económica. Profesor y director del Grupo de Investigación de Modelos Dinámicos Estocásticos de Equilibrio General de la Universidad EAFIT, Medellín, Colombia, [jabotero@eafit.edu.co].

b Magíster en Economía. Profesor y miembro del Grupo de Investigación de Modelos Dinámicos Estocásticos de Equilibrio General de la Universidad EAFIT, Medellín, Colombia, [jgarci85@eafit.edu.co]. 


\section{Desarrollo, reestructuración del gasto público y alianzas público-privadas}

Resumen. Este artículo analiza los efectos de bienestar de mejoras en la inversión en educación e infraestructura con participación público-privada, en un marco fiscal de economía pequeña y abierta para la economía colombiana. Se construye un modelo DSGE donde las familias toman decisiones de capital humano y el gobierno financia megaproyectos colocando bonos de infraestructura. Los resultados sugieren mayores efectos en el ajuste de largo plazo y mejoras del bienestar social cuando el gasto público se focaliza en sectores con mayores niveles de empleo y se introducen mejoras tecnológicas y de innovación.

Palabras clave: infraestructura, modelo DSGE, gasto público, capital humano; JEL: $\mathrm{B} 22, \mathrm{E} 27, \mathrm{E} 60$

\section{Development, restructuring of public spending and public-private partnerships}

Abstract. This paper analyzes the welfare effects of improvements in investment in education and infrastructure with public-private participation, in a fiscal framework of a small and open economy for the Colombian economy. A DSGE model is built where families make decisions on human capital and the government finances megaprojects by placing infrastructure bonds. The results suggest greater effects in the long-term adjustment and improvements in social welfare when public spending is focused on sectors with higher levels of employment and technological and innovation improvements are introduced.

Keywords: infrastructure, model DSGE, public spending, human capital; JEL: B22, E27, E60

\section{Desenvolvimento, reestruturação da despesa pública e parcerias público-privadas}

Resumo. Este trabalho analisa os efeitos do bem-estar de melhorias no investimento em educação e infraestrutura com a participação público-privada, em um contexto fiscal de economia pequena e aberta para a economia colombiana. É construído um modelo DSGE onde as famílias tomam decisões de capital humano e o governo financia megaprojetos colocando bônus de infraestrutura. Os resultados sugerem maiores efeitos no ajuste a longo prazo e melhorias do bem-estar social quando a despesa pública se focaliza em setores com maiores níveis de emprego e são introduzidas melhorias tecnológicas e de inovação.

Palavras-chaves: infraestrutura, modelo DSGE, despesa pública, capital humano; JEL: B22, E27, E60 
$\mathrm{E}^{1}$ gasto público ha sido una herramienta de alto impacto en la reducción de la pobreza y la consolidación de un crecimiento económico sostenido. En Colombia, la inversión pública en infraestructura ha disminuido de forma progresiva desde la década de 1970, debido a planes de ajuste fiscal, focalización del gasto social, ausencia de políticas sectoriales y mal manejo de los recursos públicos (De la Puente, 2012). En este sentido, la discusión no solo analiza los impactos convencionales del gasto público productivo, sino también las formas que este adopta y, en consecuencia, la manera como impacta la oferta agregada a través de la acumulación de factores productivos o de externalidades que aceleran la productividad.

Los modelos neoclásicos convencionales tienden a mostrar menores efectos debido a los ajustes en el consumo y la inversión que resultan de la conducta óptima de los agentes económicos, como respuesta anticipada a los posteriores ajustes que deben darse en las finanzas públicas para mantener su equilibrio y a las posibles acciones de política monetaria que reaccionan a los incrementos de precio generados por la expansión del gasto. En modelos en los cuales la economía se encuentra cercana a su frontera de posibilidades de producción (FPP) predomina el efecto de crowding out sobre el consumo y la inversión, haciendo que las políticas fiscales expansivas resulten ineficientes desde el punto de vista del empleo y el producto.

En los modelos neo-keynesianos se encuentran multiplicadores más elevados, cuando se incorporan inflexibilidades en precios y salarios. En estos modelos, las inflexibilidades pueden llevar a que la economía opere en niveles alejados a su FPP, y el gasto público puede generar movimientos de la economía hacia esa frontera, produciendo un impacto positivo sobre la actividad económica. Si además opera la restricción de tasas de interés cero, entonces los multiplicadores del gasto público pueden llegar a ser superiores a 1 , como muestran Woodford (2010) y Christiano et al. (2011). En efecto, una tasa de interés nominal cercana a cero puede indicar que la economía opera lejos de su FPP, y el gasto público puede cumplir un papel importante para emplear los recursos ociosos. Esto explicaría la asimetría del multiplicador en condiciones de auge y de recesión, que documentan Auerbach y Gorodnichenko (2012).

Existen por lo menos dos modalidades del gasto público que pueden afectar la FPP: el gasto en infraestructura y el gasto en educación. El primero genera externalidades positivas sobre la producción, elevando la cantidad de producto que se obtiene con una determinada dotación de capital y trabajo. Esa expansión de la FPP se refleja en 
mayores ingresos para los factores de la producción y en ingresos fiscales adicionales, que atenúan los ajustes ulteriores en impuestos o gastos futuros, responsables del crowding out sobre consumo e inversión. E1 segundo eleva la productividad del factor trabajo, en un proceso de reasignación de recursos que parece estar en la base de los episodios de crecimiento acelerado que han sido notorios en los últimos años especialmente en los países asiáticos: cuando la calidad del recurso se eleva, el crecimiento económico se apoya en la expansión de la FPP, generando círculos virtuosos que están en la base de esos episodios de crecimiento acelerado.

E1 presente trabajo busca analizar esas modalidades de gasto público en un modelo dinámico estocástico de equilibrio general (DSGE), en el cual se hacen expresas las restricciones de endeudamiento de cada agente, que se han tornado críticas para el manejo de las finanzas públicas en los últimos años. La idea es evaluar el impacto de esos tipos de gasto cuando se consideran su impacto directo y las complementariedades resultantes de las decisiones óptimas de los agentes privados, bien sea en inversión en infraestructura, a través de asociaciones público-privadas, o en la formación de capital humano.

En la primera sección se presenta la revisión de literatura; en la segunda, el modelo DSGE; la tercera se ocupa de las simulaciones y del proceso de calibración; la cuarta sección aborda el análisis de bienestar y de los multiplicadores; finalmente se presentan las conclusiones.

\section{REVISIÓN DE LITERATURA}

Existen pocos estudios que incorporen y descompongan las diferentes modalidades de financiación del gasto público en un modelo DSGE. A continuación examinamos la manera como la modelación macroeconomía ha abordado este rubro a partir de diferentes aproximaciones. Los modelos macroeconómicos neoclásicos que incorporan las decisiones del gobierno abordan diversas metodologías para analizar las distintas formas en que el sector público interviene en el funcionamiento de la economía. Un referente en estas metodologías consiste en determinar el gasto en consumo público y la manera como este afecta la utilidad de los individuos, al recaudar impuestos, hacer transferencias a los hogares y suministrar bienes públicos.

Algunos modelos de equilibrio general con consumo público son abordados por Ljungqvist y Sargent (2004), McGrattan (1994), Baxter y King (1993), Christiano y Eichenbaum (1992), Aschauer (1985), Barro (1981) y Hall (1980). Estas investigaciones incluyen el consumo de múltiples maneras, con el resultado particular de que un 
aumento del gasto público disminuye el consumo privado. Algunos resultados obtenidos en estos estudios van en contravía de la evidencia empírica, que muestra en muchos casos correlaciones positivas o nulas entre ambos.

Otras aproximaciones surgen en la década de 1970, donde se incluyó el capital público en infraestructura en la función de producción agregada. Es el caso de Arrow y Kurz (1970), Weitzman (1970) y Pestieau (1974). Dos décadas más tarde, Barro (1990) logró incluir el gasto público en la función de producción con rendimientos constantes a escala, evaluando los impactos que tiene sobre el crecimiento. Otras investigaciones teóricas introducen el concepto de capital público como variable que se deprecia periodo a periodo: Barro y Sala-i-Martin (1992), Finn (1993), Glomm y Ravikumar (1994). Cashin (1995), por su parte, desarrolla un modelo en el que considera al capital público como una variable de stock.

En cuanto a los análisis empíricos sobre el efecto del capital público, Mera (1973) estima múltiples funciones de producción del tipo Cobb-Douglas añadiendo el capital público para diferentes sectores productivos y para distintos tipos de capital para la economía japonesa. Los resultados muestran que el valor medio de la elasticidad del nivel de producción respecto al capital público es de 0,2 para el sector primario e industrial, de 0,5 para el sector de transporte y telecomunicaciones, y de 0,12 a 0,18 para el sector de servicios.

Una década más tarde, Ratner (1983) realiza un análisis similar para la economía de Estados Unidos en el periodo 1949-1973, esta vez incluyendo tres factores productivos: capital público, capital privado y trabajo. Los resultados obtenidos muestran que la elasticidad del nivel de producción respecto al capital público es de 0,058, mientras que la elasticidad en relación con el capital privado es de 0,22.

A partir de estas investigaciones ha surgido una amplia literatura empírica fundada en estimaciones de producción en las que se incluye el capital público, con resultados diferenciados. Para once países de la OCDE, Ford y Poret (1991) obtienen valores entre 0,29 y 0,66. De otra parte, Bajo y Sosvilla (1993) obtienen una elasticidad de 0,16 para la economía española. Sin embargo, otros autores encuentran resultados opuestos a los mencionados, incluso con valores no significativos. Por ejemplo, Holtz-Eakin (1992) replica el procedimiento anterior usando el mismo análisis de estimación y controlando por variables no observadas; los resultados obtenidos muestran que no existe relación entre el capital público y el nivel de producción. De otro lado, Evans y Karras (1994) estiman múltiples especificaciones de la 
función de producción para distintas definiciones de capital público, encontrando solo evidencia de gasto productivo en la educación.

Cabe destacar los estudios realizados por Mincer (1958), Porath (1967) y Neal y Rosen (2000), en los que los trabajadores deciden influir de manera directa en sus ingresos laborales mediante el gasto en escolarización o capacitación técnica, de manera que estas decisiones individuales aumentan la productividad laboral incidiendo en los salarios, la producción y el consumo. Además de analizar el gasto en educación una amplia literatura explora las variaciones de capital humano debidas a las dotaciones de riqueza y talento e incluso al tipo de institución.

La incorporación de capital humano en modelos dinámicos de largo plazo tomó un amplio lugar en la literatura: Uzawa (1965), Lucas (1988), King y Rebelo (1990), Caballé y Santos (1993), Perli y Sakellaris (1998), Pries (2001) y Krebs (2003) son algunos ejemplos de ello. En estos modelos el agente representativo identifica una función de utilidad en términos de consumo y ocio y decide cuál es el nivel óptimo de cada uno de ellos. En general, el capital humano es modelado como la acumulación de años de escolaridad mediante el gasto de los individuos en educación.

Luego de la aparición de los modelos de crecimiento endógeno en la década de 1980 y de los modelos dinámicos que incorporan choques de productividad en la dinámica del ciclo económico desarrollados por Kydland y Prescott (1982), la formación de capital humano y las decisiones de los hogares para suavizar el consumo empiezan a tomar fuerza como tema de estudio.

La principal contribución de este trabajo es analizar los efectos del gasto público en infraestructura y educación en un modelo DSGE con diversas modalidades de financiación público-privada. La restricción de ajuste fiscal del gobierno permite modelar de manera explícita el nivel de endeudamiento de una economía pequeña y abierta como la colombiana, encontrando diferentes multiplicadores del gasto público productivo y no productivo. Además, la implementación de un proceso de Markov permite considerar la transición de calificación en educación de los hogares.

\section{EL MODELO}

En esta sección se describe el modelo teórico utilizado en el análisis, tomando como referencia un régimen fiscal en países emergentes siguiendo a Botero et al. (2013). El punto de partida es un marco 
neoclásico para una economía pequeña y abierta en el que la política expansiva del gobierno interactúa con las decisiones racionales de los agentes económicos de manera que se logren estabilizar los niveles internos y externos de financiamiento público. De otra parte, modelamos las decisiones óptimas de educación público-privada mediante la introducción de procesos de Markov en las decisiones óptimas de las familias. Los planes de desarrollo del país deben buscar mecanismos para impulsar las exportaciones, de modo que se incorporan bonos en infraestructura para apalancar estos megaproyectos.

\section{Los hogares}

La economía está constituida por un continuo de hogares indexados en $j \in(0,1)$. Las preferencias de los hogares están determinadas por decisiones de consumo, trabajo calificado y no calificado, y gasto privado en educación. Así, la función de utilidad ${ }^{1}$ es:

$\operatorname{Max}_{c, n, n, i k k b} \sum_{t=0}^{\infty} \beta^{t} \frac{L_{t}}{L_{0}}\left[\operatorname{Ln}\left(c_{t}\right)+\psi_{1} \operatorname{Ln}\left(\left(1-\mu_{t}\right)-n_{t}\right)+\psi_{2} \operatorname{Ln}\left(\mu_{t}-n c_{t}\right)\right]$

donde $\beta$ es el factor de descuento; $L_{t}$ la cantidad de personas en edad de trabajar (población en edad de trabajar); $c_{t}$ el consumo per cápita; $n_{t}$ la cantidad de trabajo per cápita no calificada; $\left(1-\mu_{t}\right)$ la cantidad de población no calificada y $n c_{t}$ la cantidad de trabajo per cápita calificada. Ahora bien, la dinámica de calificación educativa que siguen las familias está definida por un proceso de Markov, de la forma $p\left(X_{n+1}=\right.$ $\left.s_{j} \mid X_{n}=s_{i}\right)=p i j \forall, n=1,2$, en el que cada uno de los estados $s_{j}$ define la matriz de transición de ser no calificado a ser calificado, así:

$\mu_{t}=\mu_{t-1}(1-t r)+\left(1-\mu_{t-1}\right) t c_{t-1}, t c_{t}: f\left(i k h_{t}, g p k h_{t}\right)$

E1 proceso de calificación de las familias se especifica con el mecanismo de transición descrito en la ecuación 2. E1 primer elemento define la probabilidad de permanecer calificados en el mercado laboral, es decir, conservar el stock de oferta laboral educada en el periodo anterior. A partir de la tasa bruta de mortalidad correspondiente al grupo etario de la población en edad de trabajar se define la tasa de

${ }^{1}$ Esta función de utilidad pertenece a la familia de las funciones CRRA (Constant Relative Risk Aversion), aumentada para incluir el ocio. Las funciones CRRA se constituyen en la manera más simple de modelar la decisión intertemporal de consumo de los agentes, generando sendas de trayectoria del consumo sensibles al precio tanto presente como futuro de los bienes consumidos. La inclusión del ocio (propuesta por Hansen, 1985) endogeniza la oferta de trabajo, haciéndola dependiente de la remuneración al trabajo, tanto presente como futuro (McCandles, 2008, p. 90). 
retiro (tr), como la proporción de individuos que pasan de ser activos a inactivos.

El segundo elemento de la matriz establece la probabilidad que tienen los miembros del hogar de mejorar sus habilidades laborales, es decir, pasar de no ser calificado a ser calificado $\left(t c_{t}\right)$. Esta está determinada por los años de educación superior finalizados por los individuos.

En cuanto a los recursos destinados a la educación de los hogares, existen dos fuentes de financiación, pública y privada. Por una parte, las familias pueden disponer una fracción de sus ingresos personales en inversión de capital humano $\left(i k h_{t}\right)$, y por el otro, el gobierno realiza gastos en educación pública en educación $\left(g p k h_{t}\right)$.

$t c_{t}=k_{\mu} i k h_{t}^{\varphi_{1}}+g p k h_{t}^{\varphi_{2}}$

De otra parte, las familias pueden adquirir bonos de infraestructura como cualquier otro activo financiero ${ }^{2}$. Estos instrumentos son emitidos por el gobierno con el propósito de conseguir grandes volúmenes de recursos a plazos que permitan ejecutar proyectos de infraestructura (de transporte, minero energéticos, de telecomunicaciones, etc.). Así, la restricción presupuestal de los hogares se define como:

$$
\begin{aligned}
& \frac{\operatorname{binf}_{t-1} R b_{t}+\operatorname{binf} f_{t-1} p a b+(1-\tau) r_{t} k_{t-1}}{1+\theta}+\left[\left(w n_{t}+w c_{t} n c_{t}(1-\tau)+e r_{t} f_{t}+\operatorname{trans}_{t}\right]+\right. \\
& {\left[c_{t}+i_{t}+i k h_{t}+i k I_{t}(1-P G I)\right]=0}
\end{aligned}
$$

donde $b_{i n f, t}$ son los bonos en infraestructura; $p a b$ corresponde a la amortización de los bonos emitidos; $R b_{t}$ es la rentabilidad de los bonos de infraestructura; $\tau$ la tasa de impuestos directos; $r_{t}$ la remuneración real al capital; $k_{t}$ el stock de capital; $w_{t}$ los salarios reales no calificados; $w c_{t}$ los salarios reales calificados; $e r_{t}$ la tasa de cambio nominal (asumimos tipo de cambio flexible y sin intervención por parte del banco central); $f_{t}$ las remesas recibidas del exterior; trans $t_{t}$ las transferencias sociales del gobierno a las familias; $i_{t}, i k h_{t}, i k I_{t}$ la inversión neta, la inversión en capital humano y la inversión en capital en infraestructura; $P G I$ la participación pública en inversión de infraestructura ${ }^{3}$.

\footnotetext{
${ }^{2}$ Este mecanismo debe cumplir con dos requisitos fundamentales: ser una alternativa viable y atractiva de financiación para el gobierno, y tener tasas y plazos atractivos para los inversionistas.

${ }^{3}$ De acuerdo con la Ley de infraestructura 1508 de 2012, los recursos de las entidades territoriales o de los fondos públicos no pueden ser superiores al $20 \%$ del presupuesto estimado de inversión.
} 


\section{Las firmas}

Las firmas operan en competencia perfecta y el capital en infraestructura entra en la función de producción como externalidad. Las decisiones de optimalidad de las firmas consisten en minimizar los factores de producción dada la función de producción:

$y_{t}=k I_{t-1}^{\vartheta}\left(\frac{K_{t-1}}{1+\theta}\right)^{\alpha_{1}}\left(z_{t} n_{t}\right)^{\alpha_{2}}\left(z_{t} n c_{t}\right)^{1-\alpha_{1}-\alpha_{2}}$

donde $y_{t}$ es la producción total que contiene bienes y servicios para la venta doméstica y del resto del mundo; $k I_{t-1}^{\vartheta}$ es el capital en infraestructura; $z_{t}$ la productividad; los parámetros $\alpha_{1}, \alpha_{2}$ corresponden a la participación del capital y el trabajo en la producción, respectivamente. La senda de capital en infraestructura evoluciona de acuerdo con:

$k I_{t}=\frac{(1-\delta i) k I_{t-1}}{1+\theta}+i k i_{t}$

siendo $\delta i$ y ikI la tasa de depreciación y la inversión en capital en infraestructura, respectivamente.

\section{Demanda de importaciones}

Suponemos un país pequeño de economía abierta que logra adquirir la cantidad de bienes que requiere la demanda interna. La demanda de importaciones sigue un proceso de minimización del gasto, de manera que no existe sustitución perfecta entre bienes importados y domésticos:

$\operatorname{Min} d_{d, m} p m_{t} m_{t}+p d_{t} d_{t}$

donde $p m_{t}$ es el precio de las importaciones en moneda nacional, $m_{t}$ las importaciones, $p d_{t}$ el precio de la producción nacional doméstica reservada al mercado nacional y $d_{t}$ las ventas de producción doméstica en el mercado nacional. Así, en este rubro la restricción corresponde a:

$c_{t}+i_{t}+i k I_{t}+i k h_{t}+g k h_{t}=b\left(\left(\omega m^{\frac{\sigma-1}{\sigma}}+(1-w)^{\frac{\sigma-1}{\sigma}}\right)^{\frac{\sigma}{\sigma-1}}\right.$

aquí, $b, \omega$ y $\sigma$ son los parámetros de escala, participación y sustitución de la función CES, respectivamente. La función de expansión correspondiente a la senda de expansión es:

$\frac{m_{t}}{d_{t}}=\left(\frac{p m_{t}(1-\omega)}{p d \omega}\right)^{-\sigma}$ 
donde el precio externo (prom), la tasa arancelaria (aran) y la tasa de cambio real (er) determinan el precio doméstico de las importaciones, así:

$p m_{t}=p w m_{t} r_{t}(1+\operatorname{aran})$

Finalmente, se define a las compras totales de los comercializadores como:

$c_{t}+i_{t}+i k I_{t}+i k h_{t}+g k h_{t}=\left(1+i v a_{t}\right)\left(p m_{t} m_{t}+p d_{t} d_{t}\right)$

\section{Demanda de exportaciones}

La interacción entre la oferta y la demanda de las exportaciones determinará los precios externos. Los bienes exportados por el país son sustitutos imperfectos de los bienes procedentes del resto del mundo, y la demanda de exportaciones se establece a partir del proceso de minimización del gasto del resto del mundo:

$\min _{x, x x} p w x_{t} x_{t}+p w_{t} x x_{t}$

donde $p w x_{t}$ es el precio de las exportaciones en moneda extranjera, $x_{t}$ las exportaciones, $p w_{t}$ el precio de los bienes que compiten con las exportaciones locales en el mercado mundial, $\mathrm{y} x x_{t}$ otros abastecedores en el mercado global. Así, la restricción de la demanda corresponde a:

$x t_{t}=b e\left(w e x_{t}^{\frac{\sigma e-1}{\sigma e}}+(1-w e) x x_{t}^{\frac{\sigma e-1}{\sigma e}}\right)^{\frac{\sigma e}{\alpha e-1}}$

donde $x t_{t}$ designa las compras totales en el mercado internacional, y be, we y $\sigma e$ indican los parámetros de escala, participación y sustitución en la función de agregación mundial, respectivamente. Análogamente, la senda de expansión es:

$\frac{x_{t}}{x x_{t}}=\left(\frac{p w x_{t}(1-\omega e)}{p w \omega w}\right)^{-\sigma e}$

\section{Oferta de exportaciones}

En este proceso, los empresarios deciden cómo asignar la producción hacia el exterior o cubrir la demanda nacional, es decir:

$\operatorname{Min}_{x, d} p x_{t} x_{t}+p d_{t} d_{t}$

La restricción entrará nuevamente como función de los parámetros de escala $b d$, participación $w d$ y sustitución $\sigma d$ de la función CET de elasticidad constante de transformación: 
$y_{t}=b d\left(w d x_{t}^{\frac{\mathrm{\sigma} d-1}{\mathrm{\sigma} d}}(1-w d) d_{t}^{\frac{\mathrm{\sigma} d-1}{\mathrm{\sigma} d}}\right)^{\frac{\mathrm{\sigma} d}{\mathrm{\sigma dd}-1}}$

Finalmente, la senda de expansión contiene el precio doméstico de las exportaciones que es función del precio en moneda extranjera $p w x_{t}$, y de la tasa de cambio $e_{t}$ :

$p x_{t}=p w x_{t} e r_{t}$

donde la venta total de las empresas se determina así:

$p y_{t} y_{t}=p x_{t} x_{t}+p d_{t} d_{t}$

\section{El gobierno}

El mecanismo de financiación del gobierno es realizado vía crédito externo, de manera que las rentas percibidas para saldar el déficit fiscal provienen de impuestos directos, impuestos indirectos y aranceles. Estos ingresos son utilizados para ejecutar sus planes nacionales de desarrollo. La restricción presupuestal se define como:

$s_{t} e r_{t}=\tau_{t}\left[\frac{r t k_{t-1}}{1+\theta}+w_{t} n_{t}+w c_{t} n c_{t}\right]+\frac{i v a_{t}\left(c_{t}+i_{t}+g_{t}+i k I_{t}+g k b_{t}\right)}{1+i v a_{t}}+\operatorname{aran}_{t} p w m_{t} e r_{t} m_{t}-$

$g_{t}-i k i_{t}-g k h_{t}-$ trans $_{t}-\frac{b_{i n f, t} R b_{t}}{1+\theta}$

donde $s_{t}$ es el saldo de deuda pública, $e r_{t}$ la tasa de cambio expresado en moneda doméstica por dólar, iva $a_{t}$ el impuesto sobre el valor añadido (impuesto indirecto) y aran $_{t}$ los ingresos fiscales por concepto de aranceles.

En cuanto al costo de endeudamiento externo $\left(r i_{t}\right)$, se incluye una prima de riesgo $(\aleph)$ que es función de la capacidad de endeudamiento del país:

$r i_{t}=\aleph\left[\frac{s_{t} e r_{t}}{P I B_{t}}\right]+r m_{t}$

donde $r m_{t}$ es la tasa libre de riesgo.

Los bonos de infraestructura que ofrece el gobierno para la ejecución de megaproyectos en infraestructura se representan como:

$b_{t}=b_{t-1}\left(1-p a b_{t}\right)+(1-P G I) i k I_{t}$

donde $p a b_{t}$ es la amortización de los bonos emitidos. 


\section{Cierre externo y fiscal}

El modelo permite ajustes de la tasa de cambio que equilibra la oferta y la demanda de divisas, las cuales son generadas por la demanda de importaciones, la oferta de divisas proveniente de exportaciones, las remesas del exterior al sector privado y el endeudamiento externo del gobierno. Igual que en Botero et al. (2013), se considera una variable de holgura para que se cumpla la ley de Walras:

walras $_{t}=p w x_{t} x_{t}+f_{t}+\left(s_{t}-\frac{s_{t-1}}{1+\theta}\left(1+r i_{t}\right)\right)-p w m_{t} m_{t}$

En cuanto al cierre fiscal, el ajuste puede realizarse mediante los tres impuestos señalados ( timp $_{i}$ ), en función de la diferencia entre la capacidad de endeudamiento en el exterior y la meta de endeudamiento, establecida así:

timp $p_{i, t}=\operatorname{timp} 0_{i}+k t i m p_{i}\left(\frac{s_{t-1} e r_{t-1}}{P I B_{t}}-m e t a\right)$ para $i=\tau, i v a$, aran

donde: $\operatorname{tim} \mathrm{P}_{i}$ es la tasa de impuestos actuales en el momento de establecer el choque, ktimp $p_{i}$ la participación de la brecha de endeudamiento que cubre los impuestos cada periodo, y meta el nivel de endeudamiento fijado por el gobierno para el periodo establecido.

\section{CALIBRACIÓN Y SIMULACIONES}

Se simularon choques estocásticos sobre la productividad, la inversión pública en infraestructura, la educación superior y el gasto público no productivo (burocrático), analizando la dinámica del ciclo económico en un modelo neoclásico contiguo a la FPP. En este contexto, el modelo propuesto ilustra el crecimiento económico de largo plazo explicado por determinantes de oferta con hogares sin restricciones de liquidez (decisiones óptimas). Las variables del presente modelo y los parámetros estructurales se calcularon a partir de las cuentas nacionales para la economía colombiana, tomando como año base 2011 (ver cuadros 1 y 2).

\section{Efectos de un choque de productividad}

Como se ilustra en la gráfica 1, la dinámica de largo plazo ante mejoras en el progreso tecnológico incrementa las rentas salariales de las familias predominando el efecto ingreso, resultado en mejoras en la inversión privada de educación y consumo privado. Ante esto, se evidencia una mayor acumulación de capital y de inversión acelerando la producción nacional. 
Cuadro 1

Parámetros del modelo

\begin{tabular}{|c|c|c|c|c|c|c|c|}
\hline$\alpha_{1}$ & 0,4046 & $p w$ & 1,0000 & $b$ & 1,9935 & $\Upsilon$ & 0,0650 \\
\hline$\alpha_{2}$ & 0,3200 & pwm & 0,9588 & $\sigma$ & 1,5000 & $i k I_{0}$ & 0,3936 \\
\hline$\theta$ & 0,0156 & $\mathrm{ma}$ & 0,0000 & $p w x 0$ & 1,0000 & $\mu_{0}$ & 0,1827 \\
\hline$\psi_{1}$ & 0,4021 & rho0 & 0,7500 & $f_{0}$ & 0,2224 & trans $_{0}$ & 1,1849 \\
\hline$\psi_{2}$ & 0,1298 & $\mathrm{rm}$ & 0,0300 & $\omega_{e}$ & 0,2262 & $t r$ & 0,1098 \\
\hline$\delta$ & 0,0976 & $\kappa$ & $-0,0669$ & $b_{e}$ & 1,4512 & $t c_{0}$ & 0,0245 \\
\hline$\delta_{1}$ & 0,0400 & meta & 0,09386 & $\sigma_{e}$ & 3,0000 & $\varphi_{1}$ & 0,8806 \\
\hline$g_{0}$ & 2,6977 & $\tau_{k}$ & 0,5000 & $\omega_{d}$ & 0,6594 & $\varphi_{2}$ & 0,1193 \\
\hline$x_{0}$ & 3,2778 & $i v a_{k}$ & 0,5000 & $b_{d}$ & 2,2186 & $g k h_{0}$ & 0,1180 \\
\hline$z_{0}$ & 30,8990 & $\operatorname{aran}_{k}$ & 1,0000 & $\sigma_{d}$ & $-2,0000$ & $p a b$ & 0,1000 \\
\hline$p x_{0}$ & 1,0000 & $\tau_{0}$ & 0,1668 & $x t_{0}$ & 1,3439 & $p g i$ & 0,2000 \\
\hline$\beta$ & 0,9438 & $i v a_{0}$ & 0,1117 & $k t_{u}$ & 0,0926 & & \\
\hline$\omega$ & 0,3075 & $\operatorname{aran}_{0}$ & 0,0429 & & & & \\
\hline
\end{tabular}

Fuente: elaboración propia.

Cuadro 2

Variables del modelo

(Logaritmos)

\begin{tabular}{|c|c|c|c|c|c|c|c|}
\hline$n$ & $-0,8996$ & $z$ & 3,4307 & $m$ & 1,29111 & trans & 0,1697 \\
\hline$n c$ & $-2,0208$ & $g$ & 0,9924 & $r f$ & $-4,3348$ & $s$ & 0,4959 \\
\hline$k$ & 3,52389 & $x t$ & 4,9007 & $d$ & 2,50862 & ri & $-3,7417$ \\
\hline$r$ & $-1,6680$ & $p w x$ & 0,0000 & $x$ & 1,18719 & $\mu$ & $-1,6995$ \\
\hline$w$ & 2,50535 & pib & 2,8619 & $p m$ & 0,00000 & $t c$ & $-3,7059$ \\
\hline$w c$ & 3,47613 & $\tau$ & $-1,7903$ & $p d$ & 0,00000 & $i k h$ & $-1,2175$ \\
\hline$c$ & 2,34328 & $i v a$ & $-2,1915$ & $p x$ & 0,00000 & $g k h$ & $-2,1363$ \\
\hline$y$ & 2,74508 & aran & -31488 & py & 0,00000 & binf & 1,0176 \\
\hline$f$ & $-1,5030$ & $i k i$ & $-0,9322$ & er & 0,00000 & $R b$ & $-2,8225$ \\
\hline$i$ & 1,33064 & $k i$ & 1,9726 & $x x$ & 4,87607 & u & 3,6527 \\
\hline
\end{tabular}

Fuente: elaboración propia.

Los avances tecnológicos, junto con la depreciación de la moneda local, impulsan la elasticidad precio de las exportaciones sobre las importaciones, registrando un incremento promedio de medio punto porcentual en las exportaciones netas a lo largo de los primeros diez periodos.

Al igual que en Solow (1957), en un modelo neoclásico de acumulación de capital con progreso tecnológico se evidencia un crecimiento económico de largo plazo con una desviación porcentual promedio del 0,86\% respecto al nivel de estado estacionario durante los primeros nueve periodos. En consecuencia, el bienestar social de los individuos, medido como incrementos de la utilidad representativa de los hogares, aumenta por los progresos tecnológicos que mejoran la educación de las familias y trasladan un mayor número de trabajadores no calificados a calificados. 
Gráfica 1

Efectos de un choque de productividad positivo

(Desviación porcentual de su estado estacionario)

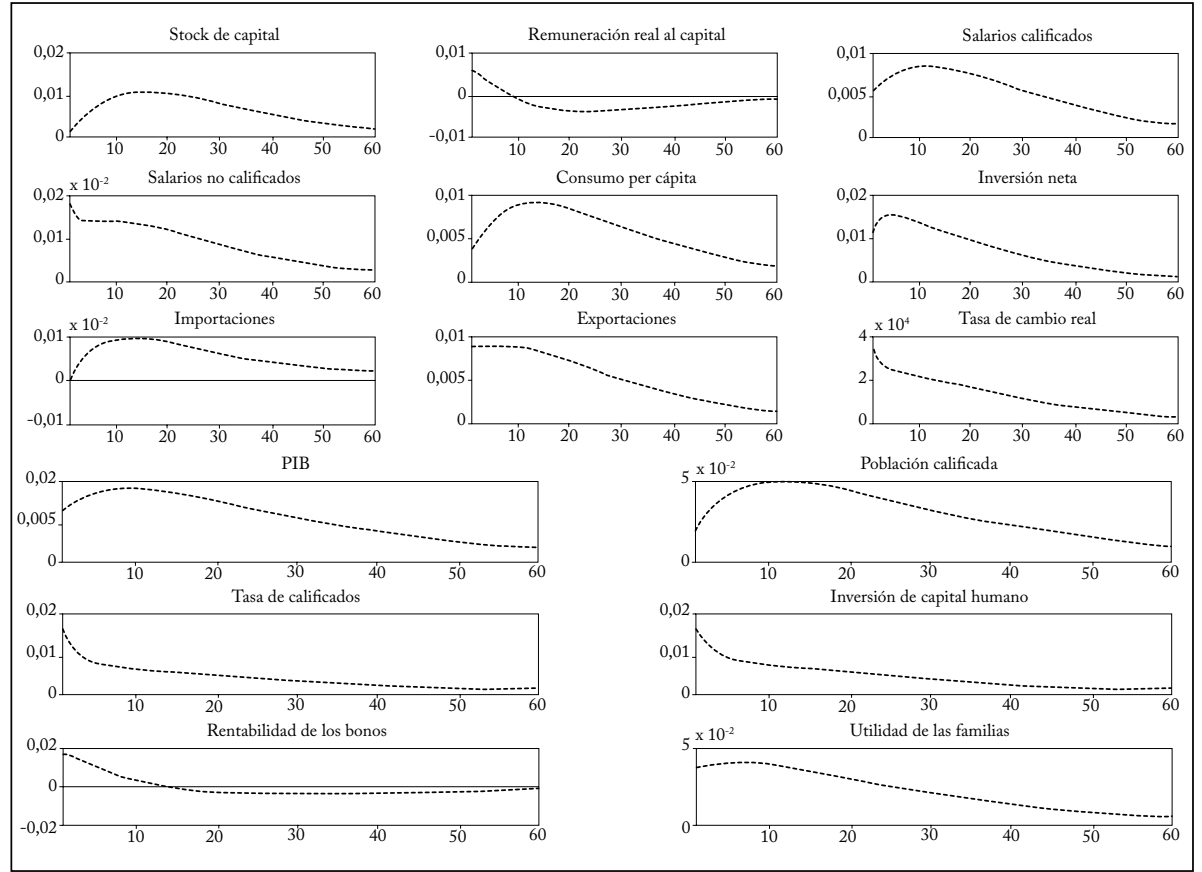

Fuente: elaboración propia.

Las nuevas oportunidades por las que atraviesa la economía frente a este escenario inducen al gobierno a captar mayores oportunidades para las familias, ofreciendo un mayor margen en la rentabilidad de los bonos en infraestructura.

\section{Efectos de un choque en inversión pública en infraestructura}

De acuerdo con la Ley 1508 de 2012, en Colombia los contratos para la ejecución de proyectos de asociación público-privada tendrán un plazo máximo de treinta años, incluidas las prórrogas. Por ello, la reacción de los principales agregados económicos y las decisiones óptimas de los agentes contenidas en este modelo reaccionan de acuerdo con la maduración y ejecución de los megaproyectos de infraestructura establecidos por el gobierno.

Como se ilustra en la gráfica 2, predomina el efecto ingreso ante incrementos salariales calificados y no calificados; estos recursos se desplazan hacia el sector de infraestructura trasladando la demanda por bonos que generen nuevas rentas para los hogares; en consecuencia, se presenta una contracción del consumo privado en bienes 
Gráfica 2

Efectos de un choque en inversión pública en infraestructura

(Desviación porcentual de su estado estacionario)

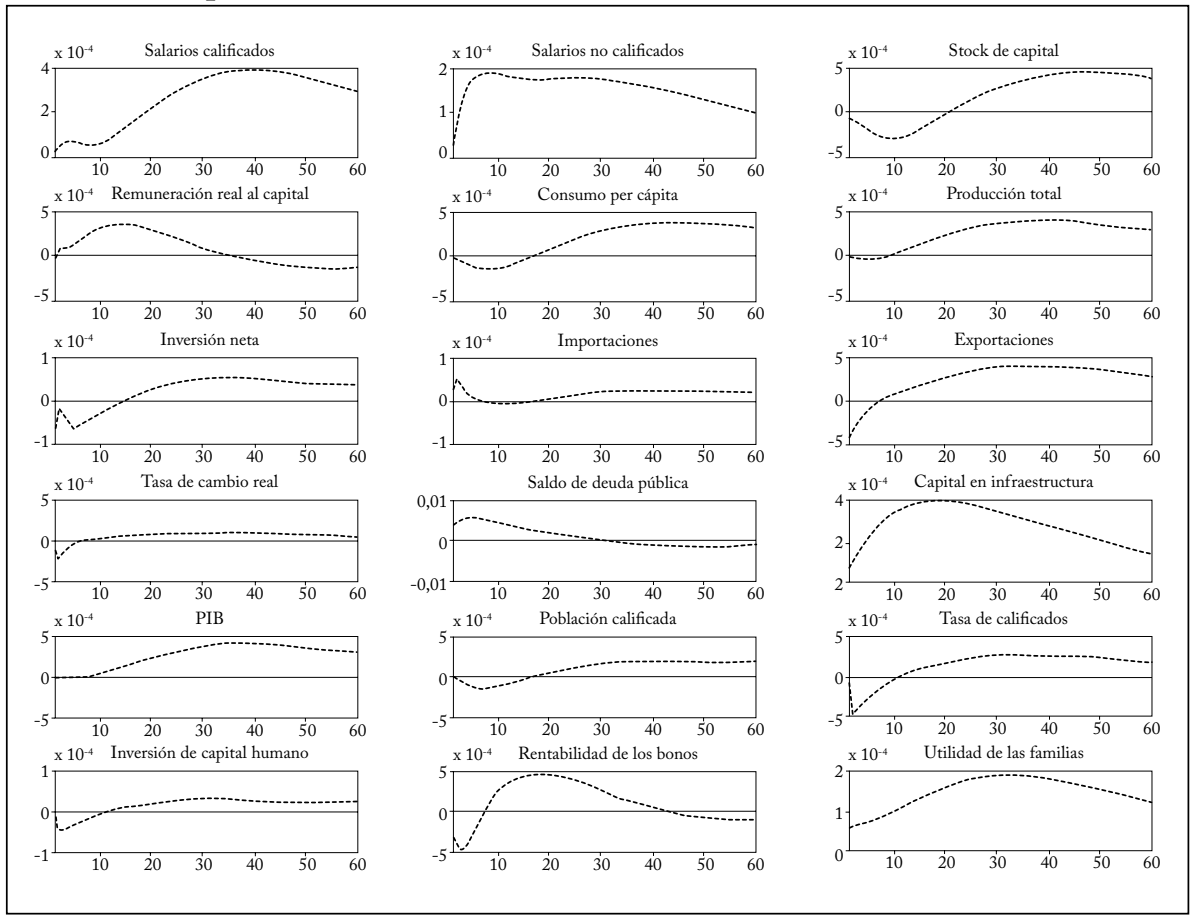

Fuente: elaboración propia.

y servicios de educación y mayor acumulación del stock de capital de infraestructura.

De acuerdo con el ranking de competitividad emitido por el Foro Económico Mundial, a lo largo de la última década Colombia ha presentado fuertes problemas en la calidad de la infraestructura en relación con sus pares latinoamericanos, convirtiéndose en una desventaja estructural que afectado el flujo comercial de bienes domésticos a los mercados internacionales.

Durante la ejecución de la inversión en nuevos proyectos de infraestructura se presenta un déficit promedio en la balanza comercial del 0,35\% respecto al nivel de estado estacionario a lo largo de los primeros seis periodos, compensado por el recaudo fiscal del gobierno mediante incrementos en impuestos indirectos. Conforme avanzan estas obras, a partir del séptimo periodo se registra un incremento promedio en las exportaciones cercano al 20\%, frente a una caída promedio en las importaciones del 13\%, para los próximos diez periodos. 
La incorporación de megaproyectos en infraestructura en los factores productivos de las firmas impacta en el crecimiento económico de largo plazo a partir del décimo periodo, con una tasa de crecimiento promedio del $0,4 \%$ respecto a su nivel de estado estacionario. Al igual que ocurre con el progreso tecnológico, la inversión pública en infraestructura consigue mejorar el bienestar social como resultado de la reducción en el nivel de precios de la producción nacional, mejoras en los términos de intercambio, rentabilidad de las familias proveniente de los bonos en infraestructura, y reasignación del gasto público hacia sectores que generan empleo y crean efectos multiplicadores en mercados con escalonamientos productivos en infraestructura.

\section{Efectos de un choque del gasto público en educación superior}

Examinemos los efectos de largo plazo en el escenario de una reasignación del gasto público. El gobierno recorta el gasto no productivo y reorienta estos recursos a la educación superior.

La construcción teórica del modelo propuesto permite capturar al menos tres canales mediante los cuales la inversión en educación afecta el crecimiento económico de largo plazo y mejor el bienestar social:

- Como resultado de la complementariedad del gasto público en educación aumenta el capital humano inherente a la oferta laboral, lo que acelera la productividad y genera mayor crecimiento económico durante la transición al largo plazo.

- Las mejoras de los procesos tecnológicos pueden dar lugar a inversiones en educación pública que estimulan la capacidad de innovación de la economía, la cual se refleja en tecnologías, procesos de producción y productos nuevos que desplazan la frontera de producción de largo plazo.

- Incluso en modelos sin restricciones de liquidez, la inversión en educación pública produce beneficios privados y sociales en la medida en que los individuos pasan a ser calificados y deciden emplearse de acuerdo con su nuevo nivel de educación; este cambio resulta en mejoras de rentas salariales, de consumo privado y de participación en bonos de infraestructura.

Así, un aumento del gasto público en educación terciaria impulsa el consumo y las rentas salariales de las familias, y reduce la inversión privada en educación en el corto plazo. La tasa de incremento promedio del número de individuos que pasan a ser calificados es 
Gráfica 3

Efectos de un choque del gasto en educación superior

(Desviación porcentual de su estado estacionario)

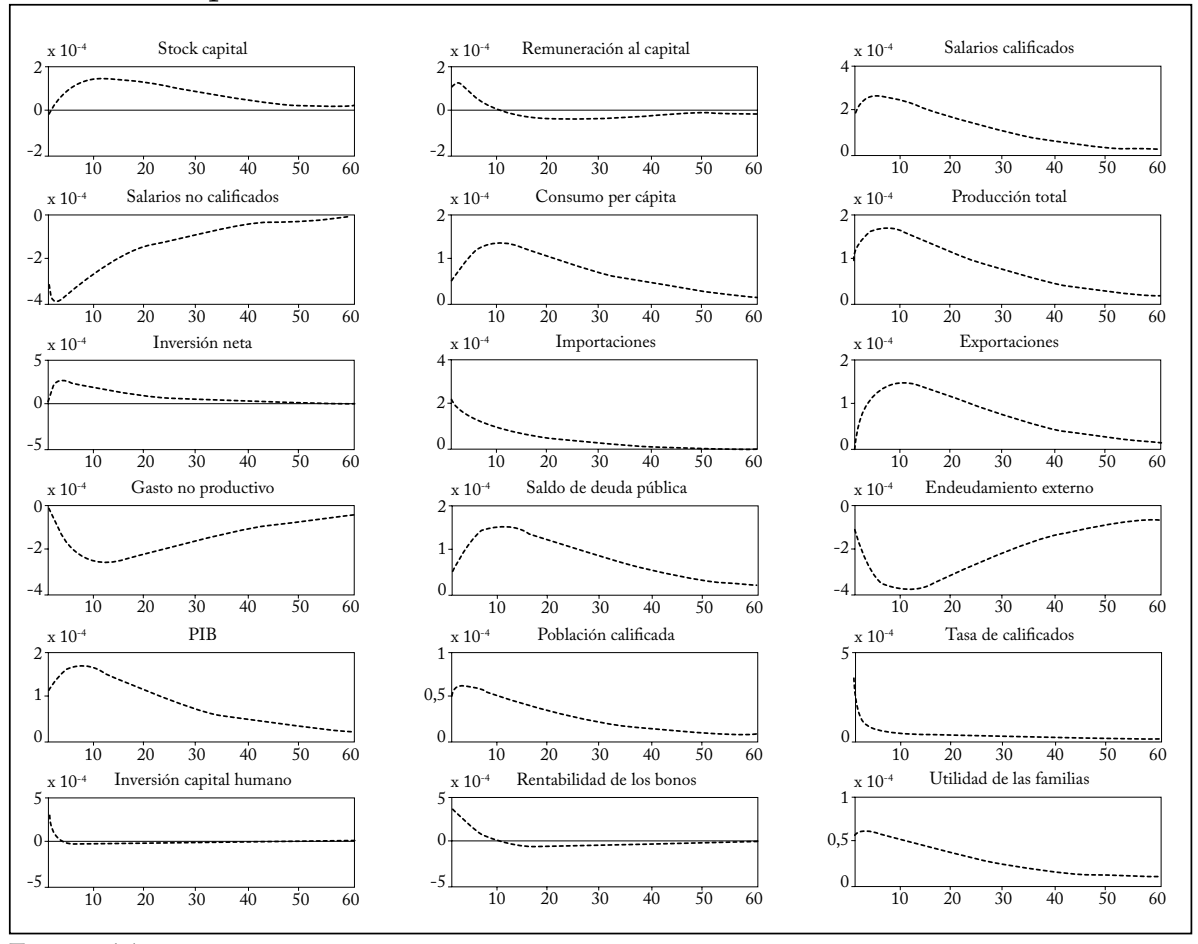

Fuente: elaboración propia.

del 5\% durante los primeros cuatro periodos, lo que eleva la tasa de calificación de los hogares durante los primeros ocho periodos. Para hacer estas inversiones el gobierno reasigna el gasto burocrático, trasladándolo a la educación superior durante los primeros ocho periodos, con incrementos transitorios promedio del $12 \%$ con respecto al nivel de estado estacionario.

El gasto público en educación superior opera como complemento de la inversión privada, y eleva el crecimiento económico de largo plazo. Durante los primeros siete periodos el crecimiento económico presenta tasas promedio del $8,3 \%$ con respecto a su nivel de estado estacionario. Los resultados obtenidos son coherentes con los de Burton (1966), Nelson y Phelps (1966), Mankiw, Romer y Weil (1992) y Benhabib y Spiegel (2005), que encuentran que la inversión en educación acompaña progresos tecnológicos que aumentan la productividad y mejoran el bienestar social. 


\section{Efectos de un choque del gasto público no productivo}

Finalmente, simulamos los efectos de largo plazo de un incremento de gasto público no productivo. Examinamos la dinámica de los principales agregados económicos si el gobierno aumentara el gasto en burocracia de las entidades centralizadas y descentralizadas.

Como ilustra la gráfica 4, el consumo se contrae a lo largo de los primeros veinte periodos a una tasa promedio del $0,18 \%$ con respecto a su nivel de estado estacionario. Este gasto, al no orientarse a sectores que generan valor agregado no se logra recuperar durante un largo intervalo de tiempo. La inversión muestra una tendencia similar, con un incremento promedio moderado durante los dos primeros periodos que oscila alrededor del $0,12 \%$ en relación con su estado inicial. De modo que la producción nacional se desacelera durante los diez primeros periodos, con tasas promedio del $0,5 \%$ con respecto a su nivel de estado estacionario; y empieza a mostrar una lenta recuperación cuando aumenta el consumo.

De otra parte, el gasto burocrático se financia con un mayor recaudo de impuestos indirectos y aumento de la deuda. A diferencia de los efectos por mejoras de productividad, inversión en infraestructura y educación superior, el incremento del gasto burocrático solo produce un ligero aumento del crecimiento durante los dos primeros periodos, a tasas promedio del $0,43 \%$ con respecto a su estado estacionario, con pérdidas de bienestar y eficiencia social.

\section{Multiplicadores del gasto}

Luego de ilustrar los efectos en el ciclo económico en diferentes escenarios de reasignación del gasto, a continuación se presentan los multiplicadores de corto, mediano y largo plazo para diferentes componentes del gasto.

Como muestra el cuadro 3, en el corto plazo el efecto de diferentes mecanismos fiscales sobre la producción resulta en multiplicadores pequeños y positivos. En modelos de economía pequeña y abierta con restricciones de gasto, la explicación es que un impulso fiscal materializado en inversión o consumo público genera mayores efectos que las transferencias; pues afecta directamente la demanda agregada alterando la renta disponible de un modo que aumenta el consumo de los hogares debido a mejoras en la productividad de los factores.

Con el paso del tiempo, los multiplicadores retornan a valores negativos. La reducción de la riqueza derivada de un mayor valor presente de los impuestos necesarios para solventar la deuda y el pago 


\section{Gráfica 4}

Efectos de un choque del gasto en burocracia (Desviación porcentual de su estado estacionario)

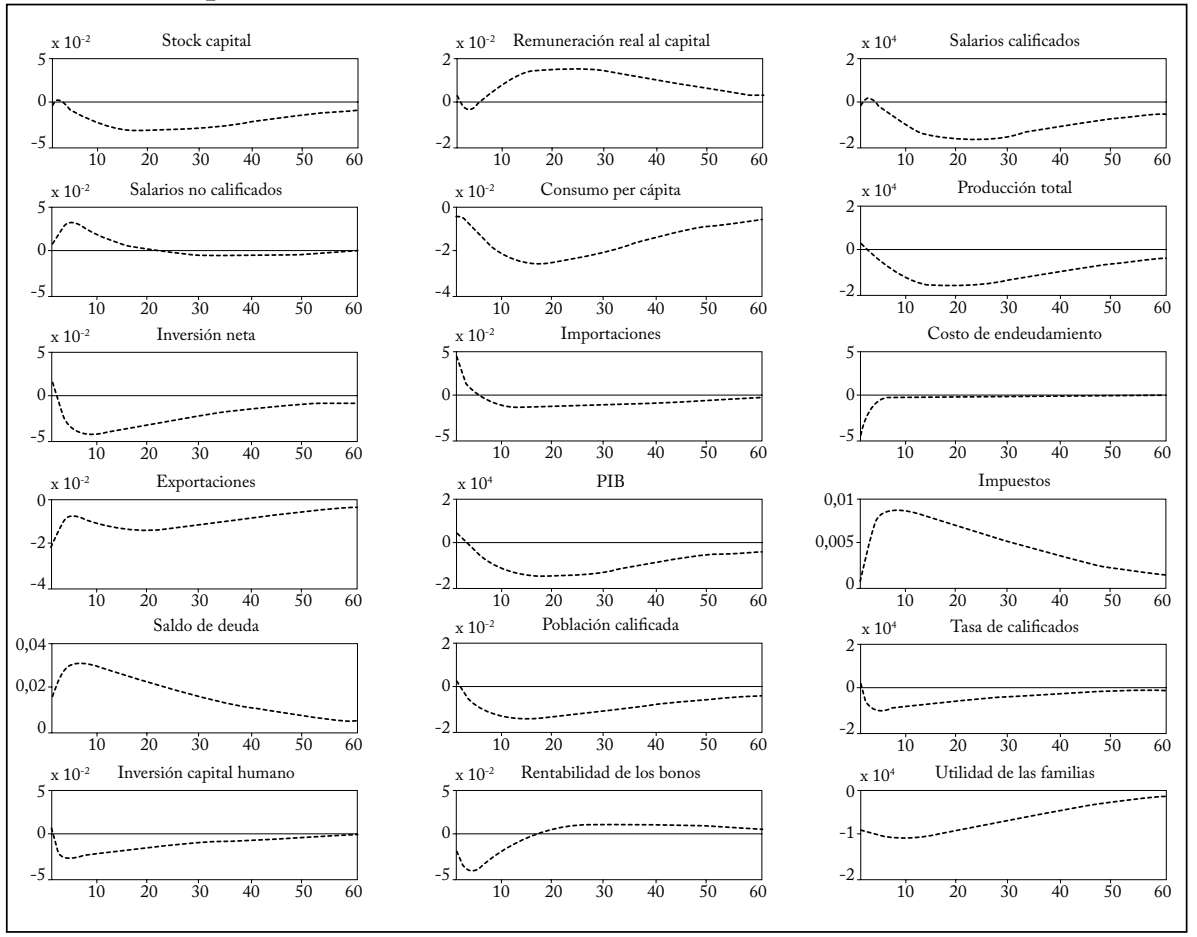

Fuente: elaboración propia.

Cuadro 3

Multiplicadores fiscales de corto, mediano y largo plazo

\begin{tabular}{lccc}
\hline \multirow{2}{*}{$\begin{array}{l}\text { Cierre del } \\
\text { modelo }\end{array}$} & \multicolumn{3}{c}{ Multiplicador de corto plazo } \\
\cline { 2 - 4 } & $\begin{array}{c}\text { Gasto no } \\
\text { productivo }\end{array}$ & $\begin{array}{c}\text { Gasto en } \\
\text { infraestructura }\end{array}$ & $\begin{array}{c}\text { Gasto en educación } \\
\text { superior }\end{array}$ \\
\hline Impuestos & 0,284 & 0,055 & 0,166 \\
Iva & 0,274 & 0,043 & 0,155 \\
Aranceles & 0,233 & $-0,003$ & 0,116 \\
\multicolumn{1}{c}{ Cierre del } & \multicolumn{2}{c}{ Multiplicador de mediano plazo } \\
modelo & Gasto no & Gasto en & Gasto en educación \\
& productivo & infraestructura & superior \\
\hline Impuestos & 0,0619 & $-0,101$ & $-0,068$ \\
Iva & 0,124 & $-0,002$ & $-0,004$ \\
Aranceles & 0,391 & 0,441 & 0,284 \\
& \multicolumn{3}{c}{ Multiplicador de largo plazo } \\
Cierre del & Gasto no & Gasto en & Gasto en educación \\
modelo & productivo & infraestructura & superior \\
& $-0,271$ & 1,335 & $-0,302$ \\
Impuestos & $-0,277$ & 1,306 & $-0,318$ \\
Iva & $-0,044$ & 0,886 & $-0,067$ \\
Aranceles & \multicolumn{3}{c}{} \\
\hline
\end{tabular}

Fuente: elaboración propia. 
de intereses por el aumento del gasto contrae la demanda privada. Además, si los impuestos no son neutrales, los efectos pueden ser aún mayores debido a que pueden afectar la oferta de capital y, por tanto, la capacidad productiva.

Los mayores efectos (multiplicadores mayores que 1) se alcanzan con la inversión en infraestructura, que logra mayores aumentos del crecimiento económico de largo plazo. Estos impactan la función de producción de las firmas como una externalidad que genera complementariedades para otros sectores productivos e impulsa los niveles de empleo, inversión y consumo de largo plazo.

\section{CONCLUSIONES}

La economía colombiana enfrenta una pérdida inminente de cuantiosas rentas provenientes del sector minero-energético. El gran desafío de la macroeconomía moderna en economías emergentes es identificar los sectores hacia los que se debe reorientar el gasto público para desplazar la frontera de producción de tal modo que se desarrollen nuevos sectores productivos. Con ese fin se propone un modelo microfundamentado con restricciones de endeudamiento expresas para cada agente, en especial para el gobierno, que reorienta el gasto público no productivo a motores institucionales que proporcionen mayores niveles de empleo y crecimiento de largo plazo: mejoras tecnológicas, educación superior e infraestructura.

Se analizan los efectos de largo plazo en una economía pequeña $\mathrm{y}$ abierta bajo un régimen fiscal con mecanismos de participación público-privados con ayuda de un modelo DSGE en el que las familias toman decisiones sobre capital humano representadas mediante un proceso de Markov y el gobierno financia megaproyectos colocando bonos de infraestructura. Los parámetros estructurales del modelo fueron calibrados utilizando datos agregados para la economía colombiana para el año 2011.

Encontramos mayores efectos sobre el crecimiento de largo plazo y la mejora del bienestar social cuando el gasto público se focaliza en sectores que concentren mayores niveles de empleo (calificado y no calificado) e implementen mejoras tecnológicas y de innovación. Igual que en un modelo neoclásico de acumulación de capital con progreso tecnológico, se logra un crecimiento económico de largo plazo con una desviación porcentual promedio del $0,86 \%$ con respecto al nivel de estado estacionario durante los primeros nueve periodos; algo similar se obtiene con mejoras en el gasto en infraestructura y 
educación superior, con incrementos promedio del $0,4 \%$ a partir del décimo periodo, e incrementos promedio del 8,3\% en los primeros siete periodos, respectivamente. En estos escenarios es importante resaltar la rápida recuperación del consumo, la inversión y la producción en el corto plazo.

Sucede lo contrario cuando se aumenta el gasto público en sectores no productivos (burocracia). El consumo se contrae en los primeros veinte periodos, a una tasa promedio del $0,18 \%$ con respecto a su nivel de estado estacionario. Al no dirigirse a sectores que generen valor agregado no se recupera en el largo plazo. La inversión presenta un moderado incremento promedio de alrededor del $0,12 \%$ con respecto a su estado inicial durante los dos primeros periodos. De modo que la producción nacional se desacelera durante los primeros diez periodos, a tasas promedio del $0,5 \%$ con respecto a su nivel de estado estacionario, y muestra una lenta recuperación cuando se recupera el consumo.

El marco propuesto permite examinar algunos rasgos de largo plazo cuando se realizan perturbaciones estocásticas en diferentes niveles de gasto sobre la frontera de posibilidades (efectos de oferta agregada). No obstante, no se consideran hogares con restricciones de liquidez, de modo que el análisis del bienestar y de los niveles de empleo se podría ampliar considerando familias heterogéneas. Es aquí donde abrimos la posibilidad de nuevas investigaciones, enlazando modelación macroeconómica dinámica y problemas de desigualdad y reasignación de recursos en economías emergentes.

\section{REFERENCIAS BIBLIOGRÁFICAS}

Arrow, K. J. y Kurz, M. (1970). Optimal growth with irreversible investment in a Ramsey model. Econometrica, 38(2), 331-344.

Aschauer, D. A. (1985). Fiscal policy and aggregate demand. The American Economic Review, 75(1), 117-127.

Auerbach, A. J. y Gorodnichenko, Y. (2012). Fiscal multipliers in recession and expansion. En A. Alesina y F. Giavazzi (eds.), Fiscal policy after the financial crisis (pp. 63-98). Chicago: University of Chicago Press.

Barro, R. J. (1981). Output effects of government purchases. Journal of Political Economy, 89(6), 1086-1121.

Barro, R. J. (1990). Government spending in a simple model of endogenous growth. Journal of Political Economy, 98(5), S103-S125.

Barro, R. J. y Sala-i-Martin, X. (1992). Public finance in models of economic growth. The Review of Economic Studies, 59(4), 645-661.

Baxter, M. y King, R. G. (1993). Fiscal policy in general equilibrium. American Economic Review, 83(3), 315-334.

Benhabib, J. y Spiegel, M. (2005). Human capital and technology diffusion. En P. Aghion y S. Durlauf (eds.), Handbook of Economic Growth, vol. 1A (pp. 935-966). Ámsterdam, Nueva York: Elsevier. 
Ben-Porath, Y. (1967). The production of human capital and the life cycle of earnings. The Journal of Political Economy, 75(4), 352-365.

Botero, J., Franco et al. (2013). Choques exógenos y política fiscal en un modelo de equilibrio general dinámico estocástico (DSGE): una aplicación para una economía emergente. Revista de Economía del Rosario, 16(1), 1-24.

Caballé, J. y Santos, M. S. (1993). On endogenous growth with physical and human capital. Journal of Political Economy, 101(6), 1042-1067.

Cashin, P. (1995). Government spending, taxes, and economic growth. IMF Staff Papers, 42(2), 237-269.

Christiano, L. J. y Eichenbaum, M. (1992). Current real-business-cycle theories and aggregate labor-market fluctuations. American Economic Review, 82(3), 430-450.

Christiano, L., Eichenbaum, M. y Rebelo, S. (2011). When is the government spending multiplier large? NBER working paper 15349.

De la Puente, M. A. (2012). Inversión pública y restricción presupuestaria en la infraestructura de transporte en Colombia: 1960-2011. Revista de Economía del Caribe, 10, 160-202.

Evans, P. y Karras, G. (1994). Is government capital productive? Evidence from a panel of seven countries. Journal of Macroeconomics, 16(2), 271-279.

Finn, M. G. (1993). Is all government capital productive? FRB Richmond Economic Quarterly, 79(4), 53-80.

Ford, R., y Poret, P. (1991). Infrastructure and private-sector productivity. OECD Economics Department, working papers 91.

Glomm, G. y Ravikumar, B. (1994). Public investment in infrastructure in a simple growth model. Journal of Economic Dynamics and Control, 18(6), 1173-1187.

Hall, R. E. (1980). Stabilization policy and capital formation. The American Economic Review, $70(2), 157-163$.

Holtz-Eakin, D. (1992). Public-sector capital and the productivity puzzle. NBER working paper 4122. Chicago: The University of Chicago Press.

King, R. G. y Rebelo, S. (1990). Public policy and economic growth: Developing neoclassical implications. NBER working paper 3338. Chicago: The University of Chicago Press.

Krebs, T. (2003). Human capital risk and economic growth. The Quarterly Journal of Economics, 118(2), 709-744.

Kydland, F. y Prescott, E. (1982). Time to build and aggregate fluctuations. Econometrica, 50(6), 1345-1370.

Ljungqvist, L. y Sargent, T. (2004). European unemployment and turbulence revisited in a matching model. Journal of the European Economic Association, 2(2-3), 456-468.

Lucas, R. (1988). On the mechanics of economic development. Journal of Monetary Economics, 22(1), 3-42.

Mankiw, N., Romer, D. y Weil, D. (1992). A contribution to the empirics of economic growth. The Quarterly Journal of Economics, 107(2), 407-437.

McCandless, G. (2008). The ABCs of RBCs. Cambridge, Mass., Londres: Harvard University Press. 
McGrattan, E. R. (1994). The macroeconomic effects of distortionary taxation. Journal of Monetary Economics, 33(3), 573-601.

Mera, K. (1973). Regional production functions and social overhead capital: An analysis of the Japanese case. Regional and Urban Economics, 3(2), 157-185.

Mincer, J. (1958). Investment in human capital and personal income distribution. Journal of Political Economy, 66(4), 281-302.

Neal, D. y Rosen, S. (2000). Theories of the distribution of earnings. En: A. Atkinson y F. Bourguignon (eds.), Handbook of Income Distribution, vol. 1 (pp. 379-427). Ámsterdam, Nueva York: Elsevier.

Nelson, R. y Phelps, E. (1966). Investment in humans, technological diffusion, and economic growth. The American Economic Review, 56(1/2), 69-75.

Perli, R. y Sakellaris, P. (1998). Human capital formation and business cycle persistence. Journal of Monetary Economics, 42(1), 67-92.

Pestieau, P. (1974). Optimal taxation and discount rate for public investment in a growth setting. Journal of Public Economics, 3(3), 217-235.

Pries, M. J. (2001). Uninsured idiosyncratic risk and human capital accumulation. Working paper, University of Maryland.

Ratner, J. B. (1983). Government capital and the production function for us private output. Economics Letters, 13(2), 213-217.

Solow, R. M. (1957). Technical change and the aggregate production function. The Review of Economics and Statistics, 39(3), 312-320.

Sosvilla-Rivero, S. y Bajo-Rubio, O. (1993). Does public capital affect private sector performance? An analysis of the Spanish case, 19641988. Economic Modelling, 10(3), 179-185.

Uhlig, H. (2010). Some fiscal calculus. American Economic Review, 100(2), 30-34.

Uzawa, H. (1965). Optimum technical change in an aggregative model of economic growth. International Economic Review, 6(1), 18-31.

Weitzman, M. L. (1970). Optimal growth with scale economies in the creation of overhead capital. The Review of Economic Studies, 37(4), 555-570.

Woodford, M. (2010). Simple analytics of the government expenditure multiplier. NBER working paper 15714. Chicago: The University of Chicago Press. 